\title{
A Review on Natural Phenomenon of Fractal Geometry
}

\author{
Ankit Garg \\ Assistant Professor \\ Amity University, Haryana \\ Manesar, Gurgaon
}

\author{
Akshat Agrawal \\ Assistant Professor \\ Amity University, Haryana \\ Manesar, Gurgaon
}

\author{
Ashish Negi \\ Associate Professor \\ GBP, Engineering college and \\ Technology \\ Pauri, Garhwal
}

\begin{abstract}
Today Fractal geometry is completely new area of research in the field of computer science and engineering. It has wide range of applications. Fractals in nature are so complicated and irregular that it is hopeless to model them by simply using classical geometry objects. Benoit Mandelbrot, the father of fractal geometry, from his book The Fractal Geometry of Nature, 1982. This paper explor various concepts of fractal i.e. fractal dimension, various techniques to generate fractal, their characteristics and their application in real life.
\end{abstract}

\section{Keywords}

Fractals, dimension, IFS

\section{INTRODUCTION}

In computer graphics we use fractal functions to create complex objects. Objects appeared in nature can be described or created by using classical geometry. People believes that the objects in nature can be created or can be described by figures such as a lines, circles, conic sections, polygons, sphere, and quadratic surfaces and so on. Many objects in nature, which are complicated and irregular can not be modeled by classical geometry. In 1918, French mathematician Gaston Julia[10] investigated the iteration process of a complex function intensively and attained a Julia set, which is a landmark in the field of fractal geometry. The Julia set defines the boundary between prisoner set and escape set[4]. The prisioner set is a collection of points inside the mendelbrot set and escape set is a collection of points out side the orbit of mendelbrot set. Julia set is the place where all of the chaotic behavior of a complex function occurs [5]. Both the Mandelbrot and Julia Set fractals require the use of complex numbers to compute the basic Mandelbrot (or Julia) set one uses the equation $\mathrm{fc}(\mathrm{Z})=\mathrm{Zn}+\mathrm{C}$, where both $\mathrm{Z}$ and $\mathrm{C}$ are complex numbers. The object Mandelbrot set given by Mandelbrot in 1979 and its relative object Julia set have become a wide and enlite area of research due to their beauty and complexity of their nature[1].

Many author have presented the paper on several " orbit traps" rendering methods to create the artistic fractal image. An orbit trap is a bounded area in the complex plane into which an orbiting point may fall[2]. Recently M.Rani and V. Kumar [3] introduced the superior Mandelbrot set using Mann iteration procedure.

"Clouds are not spheres, mountains are not cones, coastlines are not circles, and bark is not smooth, nor does lightning travel in a straight line." (Benoit Mendalbrot).

Man iteration and Ishikawa iteration process are generally used to approximate the fixed point. There is a lot of history behind Fractals even though the word fractal did not exist until the 1970s [6]. The Cantor Set discovered in 1872 by Georg Cantor
[7], Sierpinski Gasket in 1916 by Wallow Sierpinski who created a triangle and a carpet, Koch Curves in 1904 by Helge Von Koch [8], Lévy C curve in 1938 by Paul Pierre Lévy have been known for quite some time [9]. The method of function iteration analyzes the sequences of numbers $\{X n\}$ generated by the formula $X n+1=f(X n)$ where $f$ is a complex function. The colour of each point represents how quickly the values reach the escape point. Often black is used to show values that fail to escape before the iteration limit, and gradually brighter colors are used for points that escape. This gives a visual representation of how many cycles were required before reaching the escape condition.

Points bounded to origin converge to a fixed point. Fixed points can be classified in three categories:

Asymptotically stable: Means all nearby solutions converge to it.

Stable: Means all nearby solutions stay nearby.

Unstable: Means mostly all nearby solutions diverge away from the fixed point.

Formally, the fractals are categorized in two types i.e. regular (geometric) and random fractals. Regular fractals consist of large and small structures that are exact copies of each other, except in size [11]. One of the more well known regular fractals is the Koch snowflake, which is made up of small triangles added to the sides of larger triangles to an infinite degree [11] Random fractals are more apparent in nature as their small scale structures may differ in detail. It was this type of pattern that greatly influenced Mandelbrot, who gave these patterns the name fractal, from the Latin word fractus, which means a broken stone with an irregular surface.

There are two important properties of fractals are self-similarity and dimension. There are typically four techniques to generate fractals: escape-time fractals, iterated function systems, random fractals, and strange attractors [12].

The process of generating fractal image from $Z \rightarrow\left(Z^{n}+c\right)^{-1}$ is similar to the one employed for the self-squared function. By applying transformation $\mathrm{Z} \rightarrow\left(\mathrm{Z}^{\mathrm{n}}+\mathrm{c}\right)^{-1}$ again and again upto $\mathrm{N}$ times by taking $Z_{0}$ as initial value we get $Z_{1}, Z_{2}, Z_{3}, Z_{4} \ldots . Z_{n}$. In functional dynamics, we have existence of two different types of points. Points that leave the interval after a finite number are in stable set of infinity. Points that never leave the interval after any number of iterations have bounded orbits. So, an orbit is bounded if there exists a positive real number, such that the modulus of every point in the orbit is less than this number. The collection of points that are bounded, i.e. there exists $\mathbf{M}$, such that $\left|\mathbf{Q}^{\mathbf{n}}(\mathbf{Z})\right|<=\mathbf{M}$, for all $\mathrm{n}$, is called as a prisoner set while the collection of points that are in the stable set of infinity is called the escape set. 


\section{FRACTAL GEOMETRY}

A fractal can be generated by simply iterating the function again and again.

For example, if $\mathrm{f}(\mathrm{x})$ is a function and the initial value of $\mathrm{x}=\mathrm{Z}$, then , $f(Z), f(f(Z)), f(f(f(Z)))$, etc.would be the result. It may be possible that by iterating function again and again it will give larger and larger value after each iteration. For example the famous Mendelbrot's set can be generated by the following function.

$$
f(x)=Z^{\wedge} \mathbf{2}+c
$$

Suppose the initial $Z$ value is 4 . Let's say that $z 0=4$. By putting the value of $Z_{0}$ in to the function as input and iterating function again and again, we get larger and larger value as given below.

\begin{tabular}{|l|}
\hline $\mathrm{z} 1=4^{\wedge} 2+4=20$ \\
\hline $\mathrm{z} 2=20^{\wedge} 2+4=404$ \\
\hline $\mathrm{z3}=404^{\wedge} 2+4=163220$ \\
\hline $\mathrm{z} 4=163220^{\wedge} 2+4=2.66407684 \mathrm{E} 10$ \\
\hline
\end{tabular}

serpensiki triangle is can also be created by iterated function system.

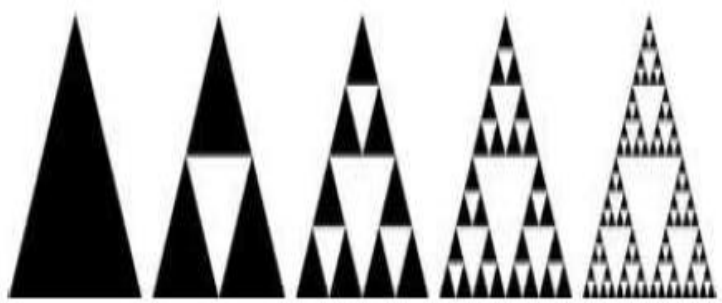

Fig 1: Construction of serpinsiki triangle using iterated function system.

\section{FRACTALS}

Many object in the nature can be created by applying the concept of classical geometry like- lines, circles, conic sections, polygons, spheres, quadratic surface and so on. There are various objects of nature which can not be modelled by applying Euclidean geometry, hence there is need to deal with such complicated and irregular object which can only be constructed by fractal geometry.

To generate such complicated object iteration process is required which is called iterated function system.

The main property in every fractal object is self similarity. Upon magnification of a Fractal, we can find subsets of it that look like the whole figure. If we zoom on a picture of a mountain again and again we still see a mountain. This is the self similarity of fractal.

\subsection{Mendalbrot set}

In a complex plane Mandelbrot set is the set of values of $c$ for which the orbit of 0 under iteration of the complex quadratic polynomial

$$
z_{n+1}=z_{n}^{2}+c
$$

remains bounded. Suppose the initial value of $Z_{0}=0$,if this function iterates again and again with a large value of $Z_{n}$, the number $C$ may or may not be part of mendalbrot set.

This function with $c=1$ gives the sequence $0,1,2,5,26, \ldots$, which tends to infinity. As this sequence is unbounded, Hence $C$ with value 1 is not an element of the Mandelbrot set.

If the value of , $C$ is -1 this function after few iteration gives the sequence $0,-1,0,-1,0, \ldots$, which is bounded, in this case $C$ belongs to the Mandelbrot set.
A time series plot of the orbit often gives more information about the fate of the orbits. Time series plots for other values of $\mathbf{c}$, are given below:

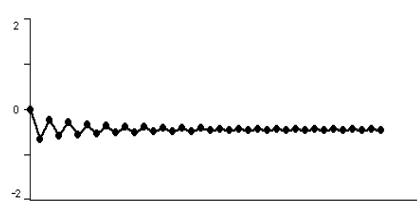

(a)

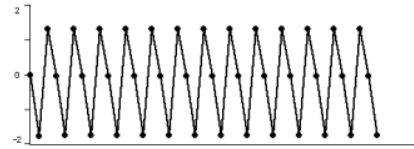

(c)

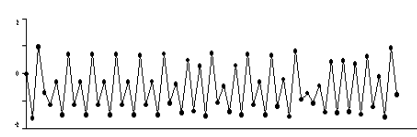

(b)

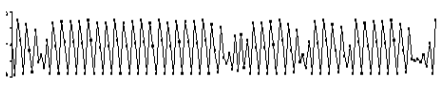

(d)
Fig 2:(a) $\mathrm{C}=-\mathbf{0 . 6 5}$ (tends to a fixed point), (b) $\mathrm{C}=-\mathbf{1 . 6}$ (chaotic behaviour), (c)-1.75 (period 3), (d)c=-1.8(Chaotic behavior close to 3-cycle, sometimes called intermittency).

Thus different points can have very different behavior under iteration by this single function $z_{n+1}=z_{n}^{2}+c$.

\subsection{Classification of fractal image}

Fractal image can be constructed as a two-dimensional array of pixels and each pixel in image can be representaed by a pair of coordinates $(\mathrm{X}, \mathrm{Y})$. The complex quantity $\mathrm{Z}$ and $\mathrm{C}$ can be represented as[18]:

$$
\begin{aligned}
& Z=Z_{x}+i Z_{x} \\
& C=C_{x}+i C_{y}
\end{aligned}
$$

Where $i=\sqrt{-1}$ and $\mathrm{Zx}$ and $\mathrm{Cx}$ are the real part and $\mathrm{Zy}$ and $\mathrm{Cy}$ are the imaginary part of $\mathrm{Z}$ and $\mathrm{C}$ respectively. Every pixel value is associated with $\left(\mathrm{C}_{\mathrm{x}}, \mathrm{C}_{\mathrm{Y}}\right)$ or $\left(\mathrm{Z}_{\mathrm{x}}, \mathrm{Z}_{\mathrm{y}}\right)$.

Fractal image can be classified as follows[18]:

Z-plane fractal: Where $(X, Y)$ is a function of $\left(Z_{x}, Z_{Y}\right)$

C-plane fractal: Where $(X, Y)$ is a function of $\left(C_{X}, C_{y}\right)$.

\section{PROPERTIES OF A FRACTAL}

\section{Self similarity}

2. Fractional dimension.

3. Fractals are non-differentiable

4. Fractal has infinite length, yet they enclose finite area.

Fractals are figures with an infinite amount of detail. After magnifying fractal it looks more complicated as they were without magnification.

\section{SELF SIMILARITY}

Fractals are self similar at any level of magnification; many things around us look the same way no matter how you magnify them. When parts of some object are similar to the entire object, we call it self-similarity.

\subsection{Perfect Self- Similarity}

According to perfect self similarity each of these fractals is composed of smaller versions of itself. When fractal with perfect self similarity is magnified, they turn out to be identical to the entire picture.

\subsection{Approximate Self-Similarity}

Sometimes, however, the object is not so perfectly self-similar. In such case, it is called approximate self-similarity. 


\section{INTRODUCTION TO DIMENSION}

A point has a dimension of 0 , a line has a dimension of 1 , a square is 2-dimensional, and a cube is 3-dimensional. Such dimension is called topological dimension.

\subsection{Similarity Dimension}

Fractal has fractional dimension. Dimension of object means how the object feels the space. Other types of dimension can be Box dimension, Hausdroff dimension.

\subsection{Method to Measure Fractal Dimension}

A Line in 1 dimensional with magnification factor 2 , will give 2 identical line segments. Let's use a variable D for dimension, e for magnification, and $\mathrm{N}$ for the number of identical shapes. Now take a 2-dimensional square and a triangle. With the magnification of 2, you get 4 identical shapes in both of them. Finally, take a 3-dimensional cube. Again, magnify it 2 times. Now, you will get 8 identical cubes. To calculate dimension of a fractal objects following formula can be used:

\section{$\mathrm{D}=\log \mathrm{N} / \log \mathrm{e}$}

To Calculate dimensions ,take a look at another fractal, called the Koch Snowflake. In it, you can see four identical snowflakes $(\mathrm{N}=4)$. Each of them is $1 / 3$ of the entire snowflake, so $\mathrm{e}=3$.

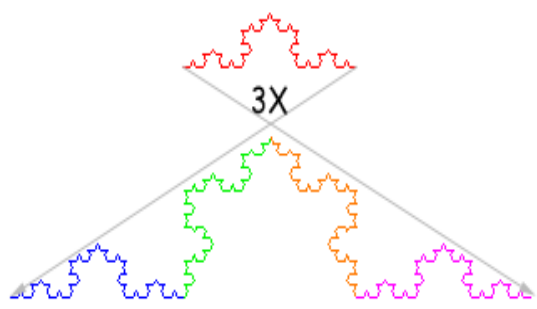

Fig 3: Fractal: Coach curve

Calculating the fractal dimension, we get: $\mathrm{D}=\log 4 / \log 3=$ 1.26. The dimension is a fraction - something you can never see in standard Euclidean geometry.

\subsubsection{Fractal Dimension-Box Counting Method}

In this method put the fractal on a sheet of graph paper, where the side of each box is size $X$. count the number of boxes which are not empty, suppose this number is K. Making the boxes smaller gives you more detail, which is the same as increasing the magnification. In fact, the magnification, e, is equal to $1 / \mathrm{X}$. In the lesson on similarity method we found that the formula for fractal dimension is $\mathrm{D}=\log \mathrm{K} / \log \mathrm{e}$. With this method we can change it to: $\mathrm{D}=\log \mathrm{K} / \log (1 / \mathrm{X})$. Making $\mathrm{h}$ smaller will make the dimension more accurate. For 3-D fractals you can do the same with cubes instead of squares, and for 1-D fractals you can use line segments.

\section{TRANSFORMATIONS}

\subsection{Affine Transformation}

Affine transformations are linear transformations. Affine transformation is composition of rotation, translations, dilations and shears. An affine transformation do not preserve angles or length. Two or more successive transformations can be applied on the image with the use of affine transformation.

A transformation $\omega: \mathbf{R}^{2} \rightarrow \mathbf{R}^{2}$ of the form

$$
\omega(x, y)=(a x+b y+e, c x+d y+f)
$$

where $a, b, c, d, e$, and $f$ are real numbers, is called a (twodimensional) affine transformation. Using equivalent notations:

$$
T\left(\begin{array}{l}
x \\
y
\end{array}\right)=s\left(\begin{array}{cc}
\cos \theta & -\sin \theta \\
\sin \theta & \cos \theta
\end{array}\right)+\left(\begin{array}{l}
e \\
f
\end{array}\right)\left(\begin{array}{l}
x \\
y
\end{array}\right)
$$

\section{TYPES OF ITERATION}

There are three basic types of iteration:

- Generator Iteration - Generating fractal by repeatedly substituting certain geometric shapes with other shapes.

- IFS Iteration - Generating fractal by repeatedly applying transformations (such as rotation and reflection) to points.

- Formula Iteration - Includes several ways of creating fractals by repeating a certain mathematical formula or several formulas[15]

\subsection{Iterated Function Systems}

In the mid 1980's, IFS's become very popular. It was Barnsley and his co-workers at Georgia Institute of Technology who first noticed the potential of IFS for applications in computer graphics. Barnsley and Sloan advertised in popular science magazines the incredible power of IFS for compressing color images at rates of over 10,000 to 1.Following are two algorithm which comes under IFS. Transformation used to generate fractals should be contractive. By applying contractive transformation on an image will create an attractor which is fractal with the same structure at any level of detail. This is the basic property of fractal. Transformation should be contractive otherwise the attractor will spread out to be infinite size. Different attractors can be generated by applying different affine transformation over the same image. Each iteration under iterated function system provides reduced copy of original one The multiple copies of original image is nothing but the result of affine transformation.

An iterated function system with probabilities consists of an IFS

$$
\left\{\mathrm{X} ; w_{1}, w_{2}, \ldots, w_{\mathrm{N}}\right\}
$$

together with an ordered set of numbers $\left\{p_{1}, p_{2}, \ldots, p_{\mathrm{N}}\right\}$, such that

$$
p_{1}+p_{2}+p_{3}+\ldots+p_{\mathrm{N}}=1 \text { and } p_{i}>0 \text { for } i=1,2, \ldots, N .
$$

The probability $p_{i}$ is associated with the transformation $w_{i}$. The nomenclature IFS with probabilities may be used as an abbreviation. The full notation for such an IFS is

$$
\left\{\mathbf{X} ; w_{1}, w_{2}, \ldots, w_{\mathrm{N}} ; p_{1}, p_{2}, \ldots, p_{\mathrm{N}}\right\} \text {. }
$$

The probabilities are related to the measure theory of IFS attractors, and play a role in the computation of images of the attractor of an IFS attractor using the random iteration algorithm. The IFS can be written as follows:

$\begin{array}{llllcc}a & b & c & d & e & f \\ 0.5 & 0 & 0 & 0.5 & 0 & 0 \\ 0.5 & 0 & 0 & 0.5 & 0 & w / 2 \\ 0.5 & 0 & 0 & 0.5 & w / 2 & w / 2\end{array}$

Fig 4: Cofficient of IFS, where $w$ is the width of the initial image.

$\begin{array}{ccccccc}a & b & c & d & e & f & p \\ 0.5 & 0 & 0 & 0.5 & 0 & 0 & 1 / 3 \\ 0.5 & 0 & 0 & 0.5 & 0 & w / 2 & 1 / 3 \\ 0.5 & 0 & 0 & 0.5 & w / 2 & w / 2 & 1 / 3\end{array}$

Fig 5: IFS with probability 


\section{ITERATING \\ FUNCTIONS COMPLEX NUMBERS \\ AND \\ WITH MANDELBROT SET}

The basic principle of generating fractals employs the iterative formula. $F(Z)=Z^{2}+$ c. Suppose the initial value of $Z$ is 0 . By putting the value of $Z$ compute $\mathbf{0}^{2}+\mathbf{c}$ which gives $\mathbf{c}$. After repeating this process we get $\left(\mathbf{c}^{2}+\mathbf{c}\right)^{2}+\mathbf{c}$. And so forth. The list of complex number will be generated in this way. If these complex numbers (called the orbit of $\mathbf{0}$ ) get larger and larger (or further and further away from the origin), then choice of $\mathbf{c}$ is NOT in the Mandelbrot set. But if this is not the case (the orbit stays "bounded"), then $\mathbf{c}$ IS in the Mandelbrot set[14].

The picture of Mandelbrot can be painted according to the following rule: Color a c-value black if $\mathrm{c}$ does not escape to infinity, it means $\mathrm{C}$ lie with in the mendalbrot set. Color a cvalue a different color if the orbit escapes to infinity. Few iteration are required for the orbit of 0 under iteration of $x^{2}+c$ to become far from the origin. Red points are followed in order by orange, yellow, green, blue, indigo, and violet. That is we use the colors drawn from the light spectrum for points that are not in the Mandelbrot set. The important thing is: red points escape fastest, while violet points take the most iterations to go far away from the origin[14].

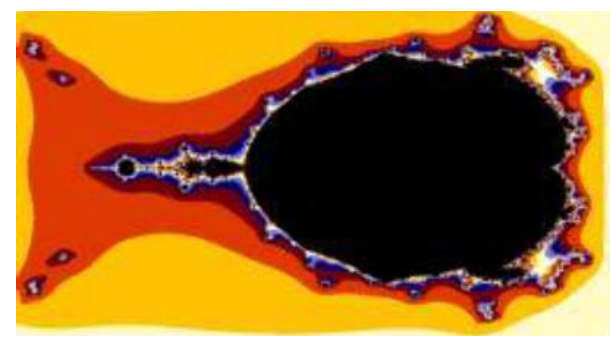

Fig 6 : orbit of mendelbrot set

The filled Julia set for $\mathbf{x}^{2}+\mathbf{c}$ is the collection of all seeds whose orbi does not escape to infinity under iteration of $\mathbf{x}^{2}+\mathbf{c}$.

Thus there is a different filled Julia set for each $\mathbf{c}$-value.

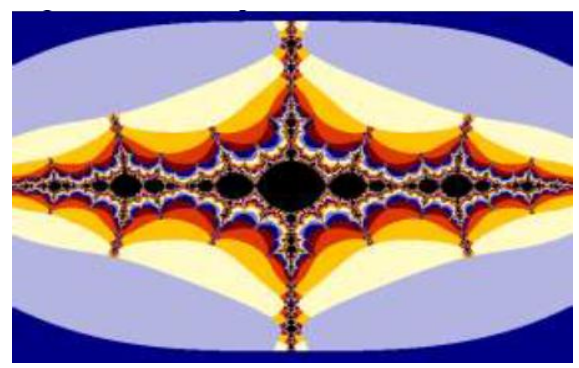

Fig 7: Corresponding Julia set of a seed inside the mendelbrot set.

\section{RELATIONSHIP BETWEEN THE MANDELBROT SET AND FILLED JULIA SETS}

Mandelbrot is a picture of the complex plane. Every point inside the Mandelbrot set represents a value for which the orbit of $\mathbf{0}$ does not escape under iteration of $\mathbf{x}^{2}+\mathbf{c}$. The shape of Julia set is depand upon the value of $c$ which may be inside the Mandelbrot set or may be out side the Mandelbrot set. If the value of $\mathbf{c}$ which is a complex number, lies in the Mandelbrot set, then the corresponding illed Julia set is connected, meaning it is just one piece. If the value of $\mathbf{c}$ lies outside the Mandelbrot set, then the filled Julia set shatters into infinitely many pieces (what is known technically as a "Cantor set" or, more popularly, "fractal dust").

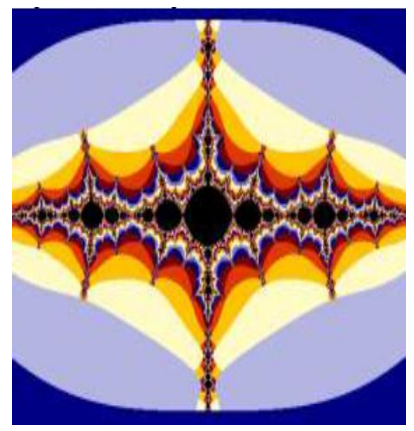

(a)

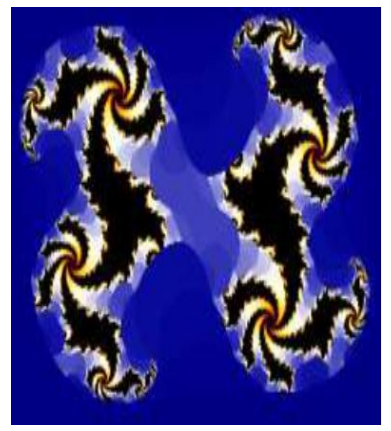

(b)
Fig 8: Julia set (a) connected Julia set (b) Disconnected Julia set

\section{ALGORITHMS UNDER IFS}

\subsection{The Deterministic Algorithm}

In this method the we start with an image and apply some affine transformation on each subset of this image and try to find out next image which should be complete subset of R2 space where image lie. After applying affine transformation again and again we get a sequence of image which should be converging at some point which will be the limit point. This limit point is nothing but an image. The apply some mapping to get an image from other image, this mapping should be contractive. For using this approach to generate fractal we require heavy amount of memory, because in each iteration we are generating some image and to store image generated by affine transformation requires large amount of memory.

Let $\{\mathbf{X} ; w 1, w 2,, w \mathrm{~N}\}$ be a hyperbolic IFS. Choose a compact set $A 0$ R2. Then compute successively $A n=W^{\circ} n(A)$ according to

$A n+1=n \mathbf{N} w j(A n)$ for $n=1,2$, . Thus construct a sequence $\{A n: n=0,1,2,3,\} \mathrm{H}(\mathrm{X})$. Then by the IFS Theorem the sequence $\{A n\}$ converges to the attractor of the IFS in the Hausdorff metric. Fractal can be generated by using Ishikawa iteration. The objective of Ishikawa iteration is to know different types of orbit to generate fractal.

\subsection{The Random Iteration Algorithm}

The random approach is different from the deterministic approach in that the initial set is a singleton point and at each level of iteration, just one of the defining affine transformations is used to calculate the next level. Which will also be a singolton point? At each level, the affine transformation is randomly selected and applied. Points are plotted, except for the early ones, and are discarded after being used to calculate the next value. The random algorithm avoids the need of a large computer memory, it is best suited for the small computers on which one point at a time can be calculated and display on a screen. On the other hand it takes thousand of dots to produce an image in this way that does not appear too skimpy. Let $\{\mathbf{X} ; w 1, w 2,, w \mathrm{~N} ; p 1, p 2,, p \mathrm{~N}\}$ be an IFS with probabilities. Choose $x 0, \mathbf{X}$ and then choose recursively, independently,

$x n\{w 1(x n-1), w 2(x n-1), w N(x n-1)\}$ for $n=1,2,3$,

where the probability of the event $x n=w i(x n-1)$ is $p i$. Thus, construct a sequence $\{x n: n=0,1,2,3,\} \mathbf{X}$. 


\section{THE COLLAGE THEOREM}

To find an IFS which results close approximation to original and attractor is called "inverse problem". Genetic algorithm have been proposed for the inverse problem. College theorem does not solve the inverse problem but it provides some better way of approaching it. The Collage Theorem gives the idea is to find an IFS whose attractor is very close approximation to the original image. The approximation or closeness of original image and attractor can be measured by using the Hausdorff metric. This result is the key to finding an IFS that can be used to compress an image. The collage theorem says that if $\mathrm{D}(\mathrm{C}(\mathrm{T}), \mathrm{T})<\mathrm{D}$ then $\mathrm{d}(\mathrm{T}, \mathrm{A})<\mathrm{d} /(1-\mathrm{S})$, where $\mathrm{C}$ is an IFS with contractivity $\mathrm{S}$ and attractor $\mathrm{A}$ and $\mathrm{T}$ is any iamge. $\mathrm{D}(\mathrm{C}(\mathrm{T}), \mathrm{T})<\mathrm{D}$ says that the distance between to point should be small because all the IFS should be contractive and the d(T,A) $<\mathrm{d} /(1-\mathrm{S})$ says the distance between orginal and attractor should be as minimum as possible or the hausdorff distance between orginal image and attractor should ne as minimum as possible. If the value of $S$ close to 1 , nothing ensures that this method provides a good approximation. Yet this was the original idea of Barnsley and most of the fractal based algorithm rely on the same approach. Quality of compression is depend upon how well the transformation manage to map the sections of the image in the range block to each domain block. Continuity Condition in collage theorem state that Small changes in the parameters of an IFS will lead to small changes in the attractor. In other words, as the coefficients of an IFS are altered, the attractor changes smoothly and without any sudden jumps.

\section{FRACTAL AND CHAOS}

The relation between fractal and chaos is very strong. Fractal geometry is used to describe the behavior of chaotic system we usually find in nature. Chaos theory is closely related to fractal theory. Many events were considered to be chaotic, unpredictable and random. A butterfly flapping its wings in a south American jungle, it is said, can lead to a hurricane in china. This is the signature of chaos theory. Chaos Theory deals with nonlinear things that are effectively impossible to predict or control, like turbulence, weather, the stock market, our brain states, and so on. Chaos is somthing which is very sensitive to initial condition. Chaotic systems are mathematically deterministic but nearly impossible to predict. Long term predictions of any system is not possible if the initial condition are known to an accurate degree. For example the weather is an example of chaotic system. In any chaotic system bifurcation means when attractor splits in two parts due to most complex calculation which is chaotic in nature. System often become chaotic when there is a feedback present. Fractal is a never ending patterns. They are created by repeating a simple process again and again in an ongoing feedback loop.To see just how far a fractal or certain conditions can diverge from its starting point, we can iterate with two initial conditions that differ by just a very small amount. The result of any IFS is fixed point which is called the attractor. As the function iterates it generates some sequence of points which finally converges to the attractor. Basin of attraction is an area which bounds the solution. Points which are not bounded by basin of attraction can approximate to infinity or further from attractor. Factals that bear this particular trait are called escape-time fractals. Points which are outside from basin of attraction are called Repellers. Some of the attractor discovered are most surprising in form. The attractor which arise for chaotic systems have been called Strange attractors. Strange attractor are differ from regular attractors in that it is impossible to tell where they will be. Fractal are related to chaos because they are complex systems that have definite properties. Fig below describe chaotic function after recursively iterate function with some value of $\mathrm{C}$.

\subsection{Escape time and Escape time Algorithm}

The escape time algorithm is employed to visualize the dynamics corresponding to the Julia sets and Mandelbrot sets [19].The value is iterated into given mathematical function to produce some list of number is called orbit. If the list of numbers (orbit) begins to take off to infinity, its ecape time is how many iteration it took before it was going to take off infinity. If the list of orbit does not take off to infinity then it is a prisoner point. Each pixel is either colored black if it is a prisoner, or given a color based on how many iterations it took to escape. It refers to some region in complex plane and typical color is used to indicate the number of iterations before iterate gets large. Escape time algorithm are employed to visualize the dynamics corresponding to the Julia sets and Mandelbrot sets. The algorithm corresponding to $\mathrm{F}(z)$ or $L(z)$, to create the escape time criteria is as follows: Select a maximum iteration bound $\mathrm{N}$, and a sense of unbounded $\mathrm{M}$. For all pixels (j, k) corresponding to the points $\mathrm{z}$ in a rectangular portion of the complex plane, do the following:

start

let $i=0$

While $z<M$ and $i<N$ do

$z=f(z)$

$i=i+1$

end while

If $i=N$, mark the pixel $(j, k)$ black,

otherwise, mark the pixel a hue that corresponds to $i$. end

\section{IMPORTANCE \& APPLICATIONS OF FRACTALS}

\subsection{Image compression}

Joint Photographic Experts Group (JPEG) and Fractal image compression are two method to compress the images. One of the most useful applications of fractals and fractal geometry is in image compression. Fractal image compression use self similarity of image to find out the redundancy in image. Fractal image compression is Resolution and device independent. Decompression by using fractal image compression is 6 times faster than JPEG. This method is good for high compression ratios. Compression ratios can be improved by taking more time during compression without any increase in decompression time or decrease in image quality.

\subsection{Fractal in architecture}

Most of the bulding created by man, has Euclidean shapes and pattern but bulding can be constructed by using fractals geometry. The reason of using fractal geometry in architecture is to mimic the patterns in Nature.

\subsection{Fractal Antenna}

Small and portable antenna such as cellular phone antenna have sensitivity to only a narrow range of frequencies. But antenna designed by fractal process have sensitivity at various frequency. Also, fractal antennas can operate efficiently at onequarter the size of more traditional designs. Several companies already are using fractals for compact, multi-frequency antennas in cellular phones and military communications hardware. 


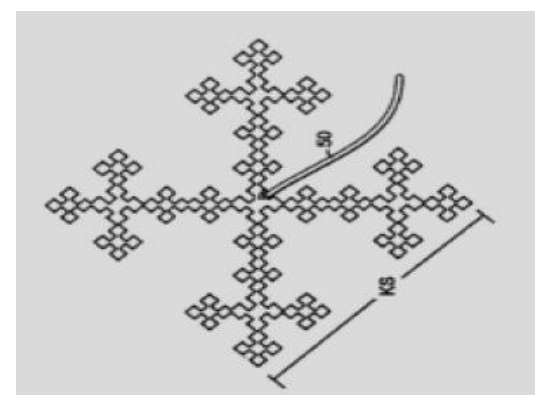

Fig 9: An example of a fractal antenna: a space-filling curve called a Minkowski Island.

\subsection{Fractal in bacteria cultures}

The spreading of bacteria can be modeled by fractals such as the diffusion fractals.

\subsection{Fractal in cables and bridges}

Fractal ideas can be used to make super-strong cables. This repetitive, fractal pattern in cables provides great strength. A steel cable is formed from a bundle of smaller cables which themselves are formed of smaller bundles, etc.

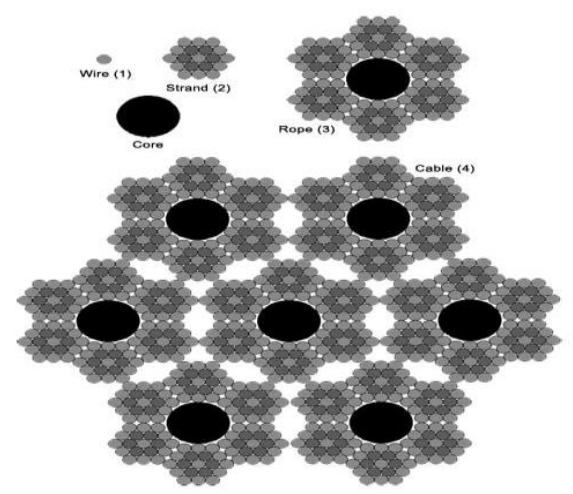

Fig 10: Use of fractal in cable construction

\subsection{Fractal in medicine}

With the use of modern medicine malfunctioning in the human body can be detected. Because human body is full of fractals, fractal math can be used to quantify, describe, diagnose and perhaps soon to help cure diseases. The fractal dimension of the lung appears to vary between healthy and sick lungs, potentially aiding in the automated detection of the disease. To diagnose Cancer, fractal analysis is helpful.

\subsection{Fractal in market analysis}

Benoit Mandelbrot introduced a new fractal theory which is helpful to analyze the market. After plotting price data of market for a month some rises and fall will be appeared in the graph. It this graph is plotted for weeak or even for a day same rises and fall will be appear. This is self similar property of fractal. It is also called Brownian self-similarity.

\subsection{Fractal in art}

The concept of Fractal can be used to create pictures which are more complicated in nature and they have the property of selfsimilarity. Mendelbrot set suggested by benoit mendalbrot is a good example of fractal science.

\subsection{Fractal in landscapes}

Fractal landscapes is a very classic application of fractals. If structure of mountainis magnified we get more and more detail at each level of magnification. The structure of small part of mountain is same as strucute of whole image. This is called self-similarity. Usually plasma fractals are used for the landscapes because they give the most realistic pictures.

\subsection{Fractal Molecules}

Fractal science can be used to study sequence of nucleotides which is called the DNA walk. The DNA walk is a graphical representation of the DNA sequence. These pattern are remarkably similar to Brownian motion.

\subsection{Fractal in plants}

Structure of plant has self-similar property. The main trunk of any plant splits into a number of branches. Each of those branches splits into smaller branches. After continuing this splitting process, a tree like structure is created. Because of numerous ways branching can be achieved geometrically, there are several ways of creating models of plants as well. One classic way of creating fractal plants is by means of 1-systems.

\subsection{Fractal in population growth}

Fractals can be used to analyze the rapid growth of population of developing countries. Last century, Thomas Malthus came with a theory in which he said that with every generation, the population increases a certain amount of times depending on the growth rate. Mathematically, if we make $r$ the percent growth rate, and $\mathrm{P}$ the population, our formula will become new $\mathrm{P}=(1+\mathrm{r}) \cdot \mathrm{P} \quad$....(1)

For example, if $r=1 / 2$ the population will increase $50 \%$, or become 1.5 times larger. According to this theory, the population will increase infinitely [18]. However, the population is really limited by natural resources, such as space and food. Let's pretend the maximum possible population the environment can hold is 1 , so $\mathrm{P}$ is a number from 0 to 1 . As the population gets closer to 1 , the growth rate is going to decrease and get close to 0 . We can achieve this by multiplying the growth rate by $(1-\mathrm{P})$. This way, as $\mathrm{P}$ is getting closer to 1 , the growth rate will be multiplied by a number that is getting close to 0 . We now determined that the growth rate should really be $\mathrm{r}(1-\mathrm{P})$. If we use it in the above formula, we get If we now do some algebra

$$
\begin{aligned}
& \text { new } P=[1+r-r P] \cdot p \\
& \text { new } P=p+r P-r P^{2} \\
& \text { new } P=(1+r) . P-r P^{2}
\end{aligned}
$$

Will now use this formula. Knowing this formula, it is easy to determine what the population becomes after a long period of time. For example, when $r$ is between 0 and 2, the population becomes 1 and stays there, no matter what it was at the beginning. When it is 2.25 , it will always end up jumping between 1.17 and 0.72 . When $\mathrm{r}$ is 2.5 , it ends up jumping between $1.22,0.54,1.16$, and 0.70 . When it is 2.5 , it ends up jumping between 8 values, and when $r$ gets higher, it jumps between 16 values. As we increase $r$, the number of these values doubles.

\section{CONCLUSION}

This paper has describes various fundamental concepts and properties of fractal geometry theory. Various methods for calculating fractal dimension has also been dicussed. Escape time algorithm is used to visualize the dynamics corresponding 
to the Julia sets and Mandelbrot sets. This paper provides discussion for basic transformations, iterated function system and other theorems which are used in fractal generation process.

\section{ACKNOWLEDGEMENTS}

I thank my guide Dr. Asish Negi, Associate professor in Department of Computer Science and Engineering, Govind Ballabh pant Engineering College, Pauri Garhwal for providing me an opportunity and resource to carry out the work.

\section{REFERENCE}

[1] Yashwant s chauhan, Rajeshri rana, Ashish negi, "MandelBar sets of Inverse Complex Function", Internationa Journal of computer applications(0975-8887), Vol 9-No.2, Nov-2010.

[2] Yashwant s chauhan, Rajeshri rana, Ashish negi, "New Julia sets of Ishikawa Iterates", International Journal of computer applications(0975-8887), Vol 7-No.13, oct-2010.

[3] M.Rani and V.Kumar, "Superior Mandelbrot set". J korea Soc, Math, edu, series, Dresearch in maths, Edu, no. 4,8(2004), 279-291.

[4] Chauhan Y. S. Rana R,and Negi A,, "Mandel-Bar Sets of Inverse Complex Function", International Journal of Computer Applications (0975-8887) Volume 9- No.2, November 2010

[5] Devaney RL. A first course in chaotic dynamical systems: theory and experiment. CO: Westview Press; 1992.

[6] Barnsley, M., 1988, "Fractals Everywhere (San Diego: Academic Press, Inc).

[7] George Cantor "On the Power of Perfect Sets of Points in Classics on Fractals" (Westview Press, 2004) pp.1123.

[8] H. Von Koch, "On a continuous curve without tangents constructible from elementary geometry", Classics on fractals (G. Edgar, ed.), Addison-Wesley, Reading, Massachusetts, 1993, pp. 25-45.

[9] Peitgen, H. O.; Jurgens, H.; Saupe, D.: "Chaos and Fractals", New frontiers of science, New York Springer,1992 984pp.

[10] B. B. Mandelbrot, The Fractal Geometry of Nature (W.H Freeman and Company, 1982).

[11] C. Pickover, "Computers, Pattern, Chaos, and Beauty", St. Martin"s Press, NewYork, 1990.

[12] Peitgen H, Jürgens H, Saupe D. Chaos and fractals: new frontiers of science. New York: Springer-Verlag; 2004

[13] M. F. Barnsley and R. Hawley, Fractals Everywhere (Boston: Academic Press Professional, 1993).

[14] http://math.bu.edu/DYSYS/explorer/tour1.html

[15] http://library.thinkquest.org/26242/full/

[16] http://people.maths.ox.ac.uk/hausel/m408k/bowens/

[17] Dr. Mamta rani, saloni, "Fractals: A Research", International journal of computer engineering and technology, ISSN 0976 - 6367(Print),Volume 4, Issue 4, July-August (2013), pp. 289-307.

[18] Yashwant s chauhan, Rajeshri rana, Ashish negi, "complex dynamics of ishikawa iterates for non integer values", International Journal of computer applications(0975-8887), Vol 9-No.2, Nov-2010.

[19] Mustapha R.; Saeki, O, "Extending generalized Fibonacci sequences and their Binet type formula", 2000 Mathematics Subject Classification. Primary 40A05; Secondary 40A25. 